\title{
Ciencia, tecnología, seguridad y defensa nacional
}

\author{
Science, Technology, Security and National Defense
}

\author{
Leoncio Molina Vásquez* \\ http://dx.doi.org/10.21503/CienciayDesarrollo.2008.v9.04
}

\section{RESUMEN}

Lo que se ha pretendido con el presente trabajo de investigación ha sido básicamente determinar la relación existente entre ciencia y tecnología y las actividades vinculadas a la defensa nacional, tratando de establecer cuáles son los mecanismos de interacción entre ambas entidades y cómo se llevan a cabo dentro de una política general de seguridad nacional. Para el efecto, se ha procedido primero a una exposición de los diferentes puntos de vista existentes en torno del tema, tanto a nivel político como filosófico y hasta económico. Finalmente, se exponen las conclusiones de la investigación, dirigidas al logro de un estado social de seguridad frente a eventuales agresiones externas, pero sin sacrificar por ello las condiciones materiales de existencia.

Palabras clave: defensa nacional, seguridad nacional.

\section{ABSTRACT}

What has been sought with the present investigation work has been basically to determine the existent relationship among science and technology and the activities linked to the national defense, being about settling down which the interaction mechanisms are among both entities and how they are carried out inside a general politics of national security. For the effect, you has proceeded first to an exhibition of the existent different points of view in lathe of the topic, so much at political level as philosophical and until economic. Finally, the conclusions of the investigation are exposed, directed to the achievement of a social state of security in front of external eventual aggressions, but without sacrificing the material conditions of existence hence.

Key words: national defense, national security.

\footnotetext{
* Docente de la Universidad Alas Peruanas, filial Arequipa.
} 


\section{INTRODUCCIÓN}

Los procesos sociales de formación del capital humano y de generación de conocimientos son complejos y prolongados; en el Perú desde 1960 se ha estado produciendo una gran paradoja en materia de desarrollo científico y tecnológico en nuestro país, porque en estas décadas han estado coexistiendo simultáneamente un esfuerzo creciente en el gasto de actividades científicas y tecnológicas, conjuntamente con una producción exigua y aún decreciente de conocimientos científicos. De acuerdo con la expansión del gasto en educación superior y en otras actividades científicas y tecnológicas, nuestro país debería situarse por encima del promedio de los países latinoamericanos; sin embargo, la producción de conocimientos según el número de patentes es mínima y presenta los niveles más bajos de Latinoamérica.

Laexplicación de la paradoja enunciada parece ser determinada por los factores siguientes:

- Escaso nivel de inversión en investigación científico-tecnológica.

- Escaso nivel de desarrollo experimental.

- Limitada calidad de la educación superior.

- La emigración.

- Las opciones ocupacionales profesionales de los jóvenes.

- Las prácticas empresariales frente a la innovación.

- La estructura productiva nacional de exportación primaria.

- Divorcio de la ciencia y tecnología de la Universidad y las Fuerzas Armadas.

Las actividades científico-tecnológicas, según la definición propuesta por la UNESCO y adoptada por el Manual de Frascarison, son aquellas actividades sistemáticas estrechamente relacionadas con la generación, producción, difusión y aplicación del conocimiento científico y técnico en todos los campos de la ciencia y la tecnología que incluyen actividades como:

1. Investigación científica y desarrollo experimental ( I+D). Comprende cualquier trabajo sistemático y creativo realizado con el fin de aumentar el caudal de conocimientos incluyendo los del hombre, la cultura, la sociedad y el uso de estos para crear nuevas aplicaciones. Se divide a su vez en investigación básica, investigación aplicada y desarrollo tecnológico experimental.

2. Educación y enseñanza científica y técnica. Se refiere a todas las actividades de educación y enseñanza superior universitaria y no universitaria en el área de las ciencias básicas y naturales, ingeniería y tecnología, más la enseñanza a nivel de postgrado, estudios especializados, capacitación y actualizaciones posteriores y formación permanente $y$ organizada de científicos e ingenieros.

3. Servicios científico-tecnológicos. Son todas aquellas actividades relacionadas con la investigación y desarrollo experimental que contribuyen a la generación, difusión y aplicación de los conocimientos científicos y tecnológicos.

PROBLEMAS DE INVESTIGACIÓN Y DESARROLLO EXPERIMENTAL

Los problemas que podemos especificar según CONCYTEC son:

a. Los recursos humanos, físicos, financieros e institucionales de investigación y desarrollo del sistema científico-tecnológico nacional son insuficientes para realizar actividades que produzcan resultados en forma regular y continua. 
b. El grado de insuficiencia de los recursos del sector investigación y del desarrollo de los sistemas no es el mismo en todos los sectores de la producción donde sus actividades se desenvuelven.

c. Las actividades de ciencia y desarrollo científico-tecnológico no están orientadas a las actividades productivas que necesita el país.

d. No hay una proporción adecuada entre las actividades de investigación básica, aplicada y de desarrollo experimental productivo; en consecuencia, existe falta de contribución a la solución delosproblemasdel país porlaescasez de proyectos de desarrollo experimental relacionados con sectores estratégicos para el desarrollo socioeconómico y la seguridad nacional.

e. El sector productivo nacional se nutre del avance tecnológico del exterior y presenta características de dependencia científicotecnológica.

\section{INNOVACIÓN TECNOLÓGICA}

Un proceso de evaluación tecnológica pasa necesariamentepordistintasetapas:investigación, invención, innovación y difusión. La innovación se considera como un conjunto de actividades relacionadas entre sí y cuyos resultados son a veces inciertos. No existe una progresión lineal entre las actividades del proceso, y frecuentemente es necesario volver a fases anteriores para resolver los problemas de puesta a punto, de tal forma que en cada etapa puede ser necesario volver a otra anterior. Conceptualizando la innovación, se dice que es la interacción entre oportunidades del mercado y el conocimiento base de la empresa y sus capacidades.

Las innovaciones tecnológicas comprenden nuevos prospectos, procesos y cambios tecnológicos significativos de productos y procesos. Una innovación ha sido implementada si se ha introducido en el mercado (innovación del producto) o si se usa dentro de un proceso de producción (innovación del proceso). Las innovaciones, por lo tanto, implican una serie de actividades científicas y tecnológicas, organizacionales, financieras y comerciales. La empresa innovadora es aquella que ha implantado productos tecnológicamente nuevos o productos y procesos significativamente mejorados durante el período analizado.

\section{INDICADORES DE PATENTES EN CIENCIA Y TECNOLOGÍA COMPARADOS INTERNACIONALMENTE}

De los indicadores científico-tecnológicos adoptaremos, como ejemplo, los indicadores de patentes, que son:

a. Gasto en actividades científicas y tecnológicas: I + D / PBI

Si bien en el gasto de ACT, el Perú registra un nivel mayor al del promedio latinoamericano, su inversión en $\mathrm{I}+\mathrm{D}$ respecto del PBI es muy reducida, lo que se evidencia al comparar este ratio con el de otros países, evidenciándose que el gasto en el Perú es exiguo en relación al PBI: 0,11 \% (USA: 2,76\%).

b. Patentes solicitadas y patentes otorgadas por países en el 2001

En el 2001, el Perú registró 980 solicitudes de patentes de invención, con una participación mayor de las solicitudes de patentes de los no residentes (Brasil: 19525 solicitudes).

\section{c. Coeficiente de invención por países}

Este coeficiente resulta de dividir el número de patentes solicitadas por los residentes por cada 10000 habitantes, en cada país. Tal indicador tiene en USA un valor de 58,41; en Brasil, de 5,30; y en el Perú, de 0,13 . 
d. Tasa de dependencia científica por países2001

Este indicador mide la relación entre las patentes solicitadas por los no residentes y las patentes solicitadas por residentes. La tasa de dependencia nos confirma, a lo largo de la serie, la gran dependencia que muestra el Perú, y todos los países latinoamericanos, de la investigación científica de los países desarrollados. En el Perú, esta tasa es muy alta en relación con el promedio latinoamericano, pues alcanza un valor de 27 , mientras en Argentina es solo de 5 .

\section{e. Tasa de autosuficiencia por países - 2001}

Este indicador mide la relación entre patentes solicitadas por residentes y el total de patentes solicitadas en cada país. La tasa de autosuficiencia en el Perú muestra valores relativamente bajos debido al exiguo número de patentes solicitadas por los residentes. Perú tiene una tasa de dependencia científica de $27(\mathrm{USA}=0)$.

\section{f. Indicadores bibliométricos - 2001}

Son las publicaciones internacionales donde el Perú ocupa los lugares menos favorables, con 228, mientras que Brasil tiene 12895 en ese mismo año.

\section{¿CONTRIBUYE I + D AL DESARROLLO DEL SISTEMA PERUANO DE SEGURIDAD Y A LA DEFENSA NACIONAL?}

Amenudosehacomentadoquelainvestigación militar en los países más avanzados contribuye decisivamente al avance de determinadas áreas de la ciencia y la tecnología, la que posteriormente redunda en beneficio de la sociedad. ¿Es este el caso de Perú? ¿Se justificarían hoy día inversiones multimillonarias en sofisticados sistemas de defensa y combate? ¿Generarán estas inversiones un beneficio neto para la sociedad?
Diversos ejemplos, que arrancan de la lejanía y se extienden hasta nuestros días, podrían emplearse como argumento de una respuesta positiva a las cuestiones anteriores. Uno de ellos, quizás el más significativo, sea el nacimiento de Internet, red global concebida como sistema de comunicación y defensa en caso de un eventual ataque nuclear en los años más duros de la Guerra Fría; otro es el hoy popular GPS, un sistema de localización creado con fines militares y del que existen, pese a mantenerse el control por parte de las Fuerzas Armadas norteamericanas, multitud de aplicaciones civiles. Pero hay más: la red de satélites que enmarañan nuestros cielos surgió de aplicaciones militares, la "guerra de las galaxias" de Ronald Reagan ha permitido mejorar ostensiblemente en los dos últimos decenios los sistemas de telecomunicaciones, y de los programas de defensa que vienen ideándose tras los atentados del 11 de septiembre en Estados Unidos se espera un avance espectacular tanto en lo que se refiere a sistemas de seguridad como de transmisión de información.

Los ejemplos citados ilustran, en el sistema norteamericano de investigación, lo que podría definirse como cadena de transmisión de investigación básica y orientada desde el sistema público, con fuertes inversiones, capaz de generar una tupida red industrial de alto valor tecnológico y de investigación aplicada. A esa red no le es ajena la transferencia de tecnología a las empresas.

¿Ocurre lo mismo en el Perú? A tenor de lo que indican las cifras, la respuesta es no, porque destina el $0,11 \%$ de su inversión pública a $\mathrm{I}+\mathrm{D}, \mathrm{y}$ a programas militares, prácticamente nada.

Los más críticos con el sistema de relación entre $\mathrm{I}+\mathrm{D}$ y la defensa nacional sostienen que la investigación de $\mathrm{C}$ y $\mathrm{T}$ militar no contribuye al desarrollo y argumentan que, pese a lo que indican las cifras, la investigación que se realiza 
en el Perú no debería ser considerada, y por tanto contabilizada, para programas militares. Frente a esta situación, los científicos y tecnólogos responsables de los distintos sistemas de $\mathrm{I}+\mathrm{D}$ peruano han argumentado:

a. Durante toda la vida republicana del Perú, la ciencia y la tecnología siempre fueron postergadas por todos los gobiernos de turno. En realidad, estas áreas estratégicas para el desarrollo casi han sido excluidas del presupuesto nacional. Este enorme error históricoha permitidoqueelPerúseencuentre completamente rezagado, junto a los países más pobres del mundo, en evidente contraste con los países que apostaron e incentivaron la investigación científica y tecnológica, los mismos que hoy se encuentran emergiendo como países desarrollados.

b. Debemos plantearnos preguntas como: ¿qué tipo de ciencia necesita el país para poder competir en la economía global?, ¿qué niveles de inversión pública serán necesarios para desarrollar sus propuestas en ciencia y tecnología?, ¿cómo establecerá las prioridades en ciencia y tecnología?, ¿qué tipo de instituciones deben ser establecidas o reformadas? Son cuestiones que deben ser respondidas de manera urgente si queremos insertar a nuestro país en la competencia tecnológica del siglo XXI.

c. El irrisorio presupuesto para investigación y desarrollo de nuestro país nos ha sometido a diferencias humillantes frente a nuestros países vecinos. Así por ejemplo, Chile, invierte 32 dólares anuales por habitante en ciencia y tecnología, en evidente contraste con los 1,50 dólares USA que invierte el Perú, lo que se refleja en el hecho de que tiene una producción científica 10 veces más baja que Chile en revistas internacionales. Esta diferencia se ve reflejada también en las cifras de exportaciones per cápita, en las que
Chile supera casi 5 veces al Perú. Si queremos alcanzar y superar a Chile, necesitamos invertir 10 veces más de lo que el Perú invierte actualmente, que es el 0,1\% del PBI anual, en ciencia y tecnología. Chile invierte más del $0,6 \%$ de su PBI.

d. El Sistema Nacional de Ciencia y Tecnología del Perú, encabezado por el CONCYTEC e integrado por los institutos como IPEN, IMARPE, IGP, INIA, INICTEL, INRENA, etc., se encuentra completamente obsoleto. Sus bajísimos niveles de producción científica expresados en el número de publicaciones y/o patentes por investigador, su falta de capacidad y liderazgo generados por los cargos políticos y de confianza, la ausencia de políticas de evaluación y rendición de cuentas, y su autismo total en relación con el desarrollo nacional demuestran que estas instituciones no cumplen los fines para los que fueron creados. Por esta razón, muchos exigen su reorganización, la cual debe consistir principalmente en la selección de sus directores ejecutivos y de sus miembros de directorio por concurso público nacional e internacional, en el establecimiento de un programa activo de reclutamiento de investigadores peruanos afincados en el extranjero, en la reducción del personal administrativo a lo estrictamente necesario, en el dominio de lo científico sobre lo burocrático, en el reequipamiento de plantas pilotos y laboratorios, y en la implementación de estrictos sistemas de evaluación para sus investigadores.

e. Es necesario que la evaluación académica y el ránking de facultades académicas a nivel nacional se realice en base a sus artículos científicos publicados en revistas arbitradas internacionales $\mathrm{y} / \mathrm{o}$ por la producción de patentes y el desmantelamiento de las altas tasas de endogamia académica presentes en nuestras universidades. 
f. Se debe buscar una verdadera política de desarrollo científico y tecnológico basada principalmente en la formación y repatriación de los recursos humanos altamente capacitados del extranjero, el establecimiento de modernos centros de excelencia bien financiados y equipados en las áreas básicas y estratégicas (como la biotecnología, la informática, las ciencias de materiales, el medio ambiente) y la implementación de la transferencia de tecnologías en las áreas estratégicas para el desarrollo nacional (agricultura, alimentación, salud, educación, industria, y medio ambiente).

g. Estamos de acuerdo con la creación del Sistema Nacional de Investigadores (SNI) -similar a los de México y Argentina-, siempre que reúna a todos los científicos activos del Perú en base a su alto grado académico y publicaciones científicas en revistas arbitradas y patentes. Este organismo permitirá elevar significativamente el nivel científico del Perú, porque el ingreso o salida de los investigadores del SNI será automáticamente regulado por su producción científica, reflejada en un número mínimo de publicaciones y/o patentes por año. De esta forma, los estímulos económicos provenientes de los fondos para la ciencia serán dirigidos única y exclusivamente a los investigadores activos que permanezcan en el SNI.

h. Existe poco conocimiento de la clase política del Perú sobre la importancia de la ciencia y tecnología en el desarrollo económico y la seguridad nacional. Se sugiere, del mismo modo que en los países desarrollados y emergentes, diseñar políticas de Estado para el desarrollo de la investigación científica y tecnológica, y su uso para el beneficio de la sociedad, la economía y la reducción de la pobreza.

i. Pensamos que es imperativo encontrar mecanismos novedosos para el financiamiento de nuestro desarrollo científico y tecnológico, a fin de abandonar nuestra dependencia adicta a los préstamos de agencias multilaterales y de cooperación internacional. Una ciencia y tecnología financiadas por los sectores productivos del país, será la mejor alternativa para ejercer la independencia al momento de establecer nuestras prioridades de desarrollo. Por ejemplo, un porcentaje de los ingresos por canon minero debería destinarse para $\mathrm{I}+\mathrm{D}$.

j. Es imperativo comprender que, debido al completo abandono de la investigación científica y tecnológica en el Perú, nuestras exportaciones tienen un escaso valor agregado y por ende un escasovalor económico. Deigual manera, la falta de investigación científica ha conducido a que nuestro país ostente los niveles más bajos en la calidad de salud, alimentación y educación en la región y en el mundo. Si continuamos en esta equivocada ruta, nuestro país estará irremediablemente condenado a la inviabilidad total y a la desgracia de mantenerse siempre como un país excluido y subdesarrollado.

Considerados los distintos puntos de vista, ¿se considera que la inversión en programas militares de investigación contribuye a la capacitación científica y tecnológica de Perú? ¿Generará esa inversión un tejido industrial? ¿Favorecerá la I+D militar el desarrollo de programas de investigación básica en Perú, así como la transferencia de tecnología y la innovación a las empresas peruanas? Si no fuera así, ¿deberíamos entender como injustificada la inversión destinada a este fin o, por el contrario, deberíamos insistir en la aportación peruana a la I+D militar? En el contexto internacional actual, ¿continúa siendo necesaria la investigación militar o tal vez debería dársele otro uso a este presupuesto? Consideramos que se debe destinar recursos para la $\mathrm{I}+\mathrm{D}$ en el campo de la defensa nacional, pues de acuerdo a las leyes de la tecnología, es allí donde se prepara parte significativa de los avances de un país. 


\section{CIENCIA, TECNOLOGÍA Y FILOSOFÍA}

$\mathrm{Al}$ respecto, existen diferentes posiciones:

- Para los humanistas, la ciencia es un medio amoral en sí mismo por el poder que genera el conocimiento y su capacidad de actuar sobre el medio, de donde se deriva una responsabilidad individual que el científico humanista resuelve en términos de elección personal, de abstencionismo o de no participación en los proyectos que considera contradictorios con su particular moral o filosofía.

- El cientificismo defiende que la actividad científica es "pura, objetiva y neutral", postulado que garantiza la ausencia de responsabilidad individual de los científicos y una separación total entre la ciencia, la cultura y la moral: los científicos son solo responsables de la ciencia en sí misma, y los tecnólogos y políticos, de sus aplicaciones. Se plantea una separación formal entre la adquisición y la aplicación de los conocimientos científicos.

- Para la actitud critica, la actividad científica es una actividad humana más y no puede disociarse de la cultura ni de la sociedad en la cual se desarrolla. La ciencia es una fuente de poder, y la responsabilidad de sus aplicaciones incumbe tanto al científico como a la sociedad. Esta línea crítica tiene su arranque claro en el período de entreguerras, en torno a la figura del cristalógrafo J. D. Bernal y de biólogos como Needham y Haldane, que teorizan el concepto de responsabilidad de los cientificos y lo llevan a la práctica a través de organizaciones pioneras como la World Federation of Scientific Workers.

La resolución de la guerra mediante la aplicación directa del poder atómico tiene su continuación en la Guerra Fría, en la cual se consagra el modelo militar como eje de la actividad científica y motor de su desarrollo en los dos bloques en que se dividía el mundo. Con más o menos éxito, los científicos críticos han actuado en múltiples frentes, intentando introducir el tema de la objeción activa con el fin de frenar la enloquecida carrera de armamentos. A partir de la actitud decidida de los científicos norteamericanos del Bulletin of Atomic Scientists en los años 40, el llamamiento Russel-Einstein de 1957 y la creación del movimiento Pugwash, se reclamay se consigue en 1974 la aprobación por las Naciones Unidas del Estatuto de los Trabajadores Científicos, donde se recogen los derechos de información y decisión de los científicos sobre el alcance y aplicaciones de sus trabajos.

El ejemplo más claro y efectivo de objeción activa contra la investigación militar es la de los científicos japoneses. Las secuelas del autoritarismo y la destrucción de su pueblo estuvieron en el origen de su decisión colectiva, al final de la guerra, de no volver a trabajar jamás para ningún tipo de aplicación militar, amparados por la Constitución impuesta por los vencedores. En varias ocasiones a lo largo de los años 60 y 70 , y más claramente en 1985, con la Iniciativa de Defensa Estratégica de Reagan, las tentaciones del gobierno nipón de replantearse el tema de la investigación militar fueron abortadas por la oposición unánime de los científicos en los principales centros de investigación del país.

Recién iniciado el siglo, tras el hundimiento del bloque de estados que hegemonizaba la URSS y la desaparición del peligro nuclear inminente, la polémica sobre la responsabilidad social de los científicos adquiere una nueva dimensión. Los problemas han cambiado, el peligro de colapso nuclear se ha alejado, pero otras amenazas muy concretas afectan a la propia supervivencia del planeta. Hace 50 años fue la lucha por la paz; hoy se suman otros desafíos: la conservación de la biodiversidad de los ecosistemas, el respeto a la diferencia y a la imperfección, la conservación 
y protección del planeta para las generaciones venideras.

La magnitud de los problemas impone que la discusión sobre la ética de los fines, la ética de los medios y la ética de las consecuencias del trabajo científico se sitúe más allá de lo individual. Y en ese sentido es necesario reeditar el llamado a la responsabilidad colectiva de los trabajadores de la ciencia.

Hans Jonas, el filósofo contemporáneo que con más vigor ha impulsado la idea de una ética de la responsabilidad, plantea esta responsabilidad como el lado ético del poder causal humano: la responsabilidad es función del poder y del saber (Jonas, 1979). Al aumentar nuestro poder causal y al aumentar nuestro poder de previsión, también aumenta nuestra responsabilidad. El filósofo llama la atención sobre las promesas y peligros de la técnica moderna y afirma que no hay sustrato ético capaz de discernir entre lo bueno y lo malo de esta nueva sociedad. En cierto modo, como afirma Paco Fernández Buey, Jonas vuelve a proponer los versos de Hölderlin: lo que puede servirnos de guia es el propio peligro que prevemos (Fernández Buey, 2000).

Pero Jonas plantea también un segundo sentido, más sustancial, de responsabilidad: una responsabilidad orientada al futuro. En un mundo tan complejo, donde no todas las consecuencias eventuales pueden preverse, debemos actuar avizorando lo imprevisible. De esta noción pueden derivarse el principio de precaución y la ética de la responsabilidad. No se trata solo de dar cuenta de nuestros actos sino de la responsabilidad de lo futuro: una situación que reclama la actuación activa para preservar lo esencial y atender al ideal de solidaridad entre generaciones (Riechmann, 1997).

Principio de precaución, ética de la responsabilidad y responsabilidad colectiva de los trabajadores de la ciencia. Estos son los términos del debate y el nudo de la cuestión que hoy afecta a ramas enteras de la ciencia, desde la biotecnología a las tecnologías de la información, pero que tiene como núcleo duro la investigación en temas militares.

\section{SEGURIDAD Y DEFENSA NACIONAL}

La Seguridad es la situación en la cual el Estado tiene garantizadas su independencia, soberanía e integridad, yla población, los derechos fundamentales establecidos en la Constitución. Esta situación contribuye a la consolidación de la paz, al desarrollo integral y a la justicia social, acciones basadas en los valores democráticos y en el respeto a los derechos humanos.

Las nuevas amenazas y otros desafíos a la seguridad constituyen problemas complejos que requieren respuestas multisectoriales, complementadas por los diferentes estamentos de la sociedad civil, todos ellos actuando en su ámbito de responsabilidad, de conformidad con el ordenamiento jurídico. Esta es la base de la integración entre el Estado y la sociedad en todos los campos de la actividad nacional, particularmente en el político, económico, social, científico-tecnológico y ecológico.

La defensa nacional es el conjunto de medidas, previsiones y acciones que el Estado genera, adopta y ejecuta en forma integral y permanente en los ámbitos externo e interno. Toda persona natural y jurídica está obligada a participar en la defensa nacional del país.

El Estado garantiza la seguridad de la nación mediante el Sistema de Seguridad y Defensa Nacional, que tiene por función preparar, ejercer y dirigir la defensa nacional en todos los campos de la actividad nacional.

La política de seguridad y defensa nacional es una política de Estado que tiene por finalidad 
orientar la selección, preparación y utilización de los medios del Estado para la obtención y mantenimientodelaSeguridadNacional, tanto en el frente externo como en el interno. Esta política está constituida por el conjunto de lineamientos generales para estructurar, coordinar y armonizar los esfuerzos de los campos de acción del Estado: defensa y desarrollo, con el objeto de hacer frente a los obstáculos, riesgos, amenazas o desafíos contra la seguridad e intereses del Estado.

Los Intereses Nacionales están constituidos por las necesidades y aspiraciones amplias y duraderas que posee la nación, y se traducen en los objetivos nacionales que, hoy presentes en el Acuerdo Nacional, vienen a ser la expresión formal de los intereses y aspiraciones.

\section{IDENTIFICACIÓN DE AMENAZAS PARA LA SEGURIDAD NACIONAL}

Se identifican las siguientes clases de amenazas:

\section{Amenazas externas}

a. Las que podrían generarse si se intentara aplicar en la subregión sudamericana doctrinas de seguridad incompatibles con la vigencia del derecho internacional.

b. Las que podrían surgir de crisis en función de la escasez de recursos naturales de valor estratégico, tales como energía, agua y alimentos.

c. El terrorismo, el narcotráfico y la delincuencia internacional.

\section{Amenazas internas}

a. Grupos terroristas y subversivos, contrarios al ordenamiento constitucional y que optan por la violencia.

b. Grupos radicales que promueven la violencia social y desbordes populares. c. Delincuencia común organizada.

d. Tráfico ilícito de drogas.

e. Corrupción.

f. Depredación del medio ambiente.

\section{LA POLÍTICA DE SEGURIDAD NACIONAL EN EL ACUERDO NACIONAL}

El esfuerzo por interpretar los intereses y aspiraciones de la nación ha sido materializado en el Acuerdo Nacional del año 2002, con la participación de representantes deorganizaciones de la sociedad civil y del Estado, quienes han señalado y definido los siguientes objetivos:

\section{- Democracia y estado de derecho}

El estado de derechoy la democracia representativa son garantía del imperio de la justicia y de la vigencia de los derechos fundamentales, así como un aspecto esencial conducente a lograr la paz y el desarrollo del país.

\section{- Equidad y justicia social}

El desarrollo humano integral, la superación de la pobreza y la igualdad de acceso a las oportunidades para todos los peruanos, sin ningún tipo de discriminación, constituyen el eje principal de la acción del Estado.

\section{- Competitividad del país}

Para lograr el desarrollo humano y solidario del país, el Estado adoptará una política económica sustentada en los principios de la economía social de mercado, reafirmando su rol promotor, regulador, solidario y subsidiario en la actividad empresarial.

- Estado eficiente, transparente y descentralizado

Consolidar un Estado eficiente, transparente y descentralizado al servicio de las personas como sujetos de derechos y obligaciones. 
A partir de estos objetivos se diseñaron 31 políticas de Estado. La novena, que corresponde a la Seguridad Nacional, establece:

"Nos comprometemos a mantener una política de seguridad nacional que garantice la independencia, soberanía, integridad territorial y la salvaguarda de los intereses nacionales. Consideramos que esta es una tarea que involucra a la sociedad en su conjunto, a los organismos de conducción del Estado, en especial a las Fuerzas Armadas, en el marco de la Constitución y las leyes. En tal sentido, nos comprometemos a prevenir y afrontar cualquier amenaza externa o interna que ponga en peligro la paz social, la seguridad integral y el bienestar general".

Con este objeto, el Estado:

a. Fomentará la participación activa de la sociedad, en el logro de objetivos de la política de seguridad y defensa nacional.

b. Garantizará la plena operatividad de las Fuerzas Armadas orientadas a la disuasión, defensa y prevención de conflictos, así como al mantenimiento de la paz.

c. Impulsará la enseñanza de los conceptos básicos de seguridad y defensa nacional en todos los niveles del sistema educativo nacional.

d. Fomentará la participación activa en la protección dela Antártica, el medioambiente, el desarrollo de la Amazonía y la integración nacional.

e. Mantendrá una estrecha coordinación entre el Sistema de Seguridad y Defensa Nacional y la política exterior para la definición y defensa de los intereses permanentes del Estado.

Otras políticas que tienen relación con la Seguridad Nacional son:

- Primera: Fortalecimiento del régimen democrático y del Estado de Derecho.
- Tercera: Afirmación de la identidad nacional.

- Sexta: Política exterior para la paz, la democracia, el desarrollo y la integración.

- Séptima: Erradicación de la violencia y fortalecimiento del civismo y de la seguridad ciudadana.

- Vigésimo quinta: Cautelar la institucionalidad de las Fuerzas Armadas y su servicio a la democracia.

- Vigésimo séptima: Erradicación de la producción, el tráfico y el consumo ilegal de drogas.

- Vigésimo octava: Plena vigencia de la Constitución y de los derechos humanos. Acceso a la justicia e independencia judicial.

- Trigésima: Eliminación del terrorismo y afirmación de la reconciliación nacional.

\section{OBJETIVOS ECONÓMICO- \\ ESTRATÉGICOS PARA LA SEGURIDAD NACIONAL}

De los objetivos y políticas del Estado, nos interesan los referentes a la economía, a la ciencia y a la tecnología.

\section{Objetivo 1}

Deben buscarse las condiciones económicoestratégicas que aseguren la paz, la integración y la prosperidad.

\section{Politicas}

a. Ocupar el territorio nacional siguiendo criterios estratégicos de desarrollo y seguridad.

b. Procurar el abastecimiento para satisfacer las necesidades básicas de la población en casos de conflictos o desastres.

c. Impulsar la investigación y el desarrollo tecnológico, particularmente en las áreas de informática y comunicaciones. 
d. Promover el desarrollo científicotecnológico orientado a la satisfacción de los requerimientos militares.

e. Promover el desarrollo científico-tecnológico con participación de la universidad peruana y de la empresa privada nacional.

f. Desarrollar una Flota Mercante Nacional.

g. Desarrollar la Reserva Aérea Nacional.

h. Fomentar la inversión de capitales nacionales en el Perú y el extranjero.

i. Generar la infraestructura necesaria para atraer el comercio de los países hacia puertos peruanos.

j. Preservar el ambiente en general y la biodiversidad en particular, en función de los intereses nacionales.

k. Proteger a la población ante los desastres naturales y sociales.

1. Participar activamente en los procesos de integración en los ámbitos subregional y regional.

\section{Objetivo 2}

Fortalecimiento de la conciencia e identidad nacional.

\section{Politicas}

a. Fortalecer el orgullo y la identidad nacional.

b. Crear conciencia de seguridad y defensa, impulsando y difundiendo la enseñanza de estos conceptos.

c. Fomentar la educación cívico-patriótica en democracia, la cultura de paz y su oposición a la violencia organizada.

\section{Objetivo 3}

Protección y promoción de los intereses nacionales en el ámbito internacional.

\section{Políticas}

a. Participar en la toma de decisiones que afecten los intereses nacionales, así como en foros de seguridad y defensa, a nivel mundial, hemisférico y regional.

b. Participar en la lucha contra el terrorismo internacional y el crimen organizado transnacional.

c. Contribuir a la paz mundial, hemisférica y regional en concordancia con el Derecho Internacional y los intereses nacionales.

d. Fortalecer y respaldar la diplomacia peruana, con un Poder Nacional que promueva y defienda los intereses nacionales.

e. Crear conciencia antártica y potenciar la presencia del Perú en ese continente.

f. Contribuir a la creación de un sistema de seguridad sudamericano.

g. Organizar a las colonias peruanas en los países de interés, afianzar su identidad y orientarlas en provecho de los intereses nacionales.

h. Incrementar las medidas de fomento de la confianza mutua con los países vecinos.

\section{LA VISIÓN MULTIDIMENSIONAL DE LA SEGURIDAD}

El 28 de octubre del año 2003, en la ciudad de México, se llevó a cabo la Conferencia Especial sobre Seguridad. Esta conferencia tuvo como antecedente inmediato la Declaración de Bridgetown del año 2002, en la que se reconoció por primera vez "que las amenazas, preocupaciones, y otros desafíos a la seguridad en el Hemisferio son de naturaleza diversa y alcance multidimensional y que el concepto y enfoques tradicionales (seguridad colectiva) deben ampliarse para abarcar amenazas nuevas y no tradicionales, que incluyen aspectos políticos, económicos, sociales, de salud y medioambientales". 
El principal acuerdo señala:

"Nuestra concepción de la seguridad en el Hemisferio es de alcance multidimensional, incluye las amenazas tradicionales y las nuevas amenazas, preocupaciones y otros desafíos a la seguridad delos Estados del hemisferio, incorpora las prioridades de cada Estado, contribuye a la consolidación de la paz, al desarrollo integral y a la justicia social, y se basa en valores democráticos, el respeto, la promoción y defensa de los derechos humanos, la solidaridad, la cooperación y el respeto a la soberanía nacional".

Esta concepción multidimensional de la seguridad lleva implícita el reconocimiento de:

- La necesidad de la cooperación entre los Estados.

- Reconoce la existencia de amenazas comunes.

- Propugna la paz.

- Reconoce valores democráticos.

- Reconoce los derechos humanos.

- Deja de lado la relación de competencia y la búsqueda del equilibrio estratégico-militar.

Se añade que esta concepción multidimensional se sostiene en los siguientes valores:

a. Cada Estado tiene derecho soberano a identificar sus prioridades nacionales de seguridad y definir sus estrategias.

b. La democracia representativa es una condición indispensable para la estabilidad, la paz y el desarrollo de los Estados del hemisferio.

c. El respeto de los derechos humanos y las libertades fundamentales y la buena gestión gubernamental son esenciales para la estabilidad, la paz y el desarrollo político, económico y social de los Estados. d. La subordinación constitucional de todas las instituciones del Estado a la autoridad civil legalmente constituida y el respeto al estado de derecho.

e. El fundamentoy la razón de ser de la seguridad es la protección de la persona humana.

f. La educación para la paz y la promoción de la cultura democrática.

g. La justicia y el desarrollo humano son necesarios para la estabilidad de cada Estado del continente.

h. Se reafirma la importancia de la participación de la mujer en todos los campos para la promoción de la paz y la seguridad.

i. Las amenazas, preocupaciones y otros desafíos a la seguridad en el hemisferio son de naturaleza diversa y de alcance multidimensional.

j. Las amenazas tradicionales a la seguridad y sus mecanismos para enfrentarlas siguen siendo importantes.

k. Las nuevas amenazas, preocupaciones y otros desafíos a la seguridad son problemas intersectoriales que requieren de respuestas múltiples de las distintas organizaciones.

1. La arquitectura de seguridad debe ser flexible y contemplar las particularidades de cada región y de cada Estado.

m. La seguridad de los Estados del Hemisferio se ve afectada por amenazas tradicionales como por nuevas amenazas de diversa naturaleza que deben ser tomadas en cuenta.

- El terrorismo, la delincuencia organizada transnacional, las drogas, la corrupción, el tráfico ilícito de armas, el lavado de dinero y las conexiones entre ellos.

- La pobreza extrema y la exclusión social.

- Los desastres de la naturaleza y los de origen humano, el VIH/SIDA y otras enfermedades y el deterioro del medio ambiente. 
- La trata de personas.

- Los ataques a la seguridad cibernética.

- Daños por el transporte de material potencialmente peligroso.

- Posibilidad de acceso a la posesión de armas de destrucción masiva por terroristas.

Estas nuevas dimensiones de la seguridad van más allá de la visión militar de la seguridad circunscrita al territorio y a la soberanía principalmente.

\section{¿QUÉ DEFENDEMOS LOS PERUANOS?}

Existen amenazas a las que, a lo largo de su historia, el Perú ha tenido que hacer frente. Algunas de ellas provenían del exterior y otras surgieron en el interior del país. Se requiere, pues, que el ciudadano conozca inicialmente lo que tiene que defender y las razones por las cuales tiene que hacerlo, porque claro está que las personas solo defienden lo que conocen, lo que sienten que les pertenece y porque admiten que aquello que se defiende constituye la base de su desarrollo presente y futuro.

Todo peruano civil o militar, persona natural o jurídica, tiene por tanto que plantearse las siguientes preguntas:

- ¿Quién amenaza al Estado y a la sociedad peruana?

- ¿En qué circunstancias se produce esa amenaza?

- ¿Qué pretenden quienes nos amenazan?

- ¿Cómo intentarán ejecutar dichas amenazas?

Para poder dar respuesta a las preguntas arriba presentadas, tenemos que conocer, en primer término, lo que defendemos los peruanos.
Solo conociendo lo que defendemos, las razones por las cuales debemos hacerlo y la propia toma de conciencia de lo que defendemos, sabremos asumir los roles para la defensa del Estado y la sociedad peruana.

Los peruanos, en general:

1. Defendemos la Constitución, porque es el instrumento jurídico-normativo que regula la vida de todos los peruanos y establece los deberes y derechos que cada peruano debe respetar y cumplir. Además, la Constitución define un modelo de sociedad que ha sido aceptado por la colectividad nacional. En este sentido, citaremos algunas de las principales normas que precisan el modelo de sociedad que la Constitución ha previsto:

a) El artículo $43^{\circ}$ indica que el Estado es uno solo e indivisible y el Perú es una República Democrática, cuya renovación del poder se efectúa a través del voto popular.

b) Con relación a la forma en que se sostiene la nación, el artículo $58^{\circ}$ señala que la iniciativa privada es libre y el Estado actúa principalmente en las áreas de promoción del empleo, la salud, educación, seguridad, servicios públicos e infraestructura.

2. Defendemos las instituciones, porque una sociedad se desenvuelve a través de instituciones como el gobierno, la familia, las Fuerzas Armadas, la propiedad, el matrimonio; es decir, la Constitución de partida de nacimiento a todas las instituciones que permiten la convivencia entre los peruanos. Sobre el particular, en los artículos $90^{\circ}, 110^{\circ}$ y $138^{\circ}$ la Constitución señala que para los efectos del gobierno, el Estado se divide en tres poderes: Ejecutivo, Legislativo y Judicial. En el caso del Poder Ejecutivo y Legislativo, la duración de su mandato es de cinco años y se logra a través del voto popular. 
En el caso del Poder judicial, sus autoridades son nombradas conforme a ley.

3. Defendemos el legado histórico y las tradiciones nacionales, porque a través de los hechos ocurridos en el pasado, los diversos personajes que vivieron en cada una de las etapas de la historia nacional, los hombres ilustres y los héroes nacionales, el pais adquiere su identidad y consolida su conciencia nacional, la que resulta vital para la defensa del Estado y las Instituciones de quienes los amenacen en el Frente Interno.

4. Defendemos los recursos naturales, porque son la fuente y la base del desarrollo nacional, del presente y del futuro. Son estos recursos y la explotación racional de los mismos los que nos permitirán alcanzar el bienestar al que cada peruano tiene derecho.

5. Defendemos la salud física y mental de la población, porque la población o los habitantes de un país son la razón de ser de las preocupaciones del Estado. El Estado, en este sentido, estaría obligado a brindar y garantizar una situación de tranquilidad y paz social que permita que cada ciudadano pueda realizarse como persona. El Estado igualmente debe garantizar y proteger la salud física y mental de todos sus pobladores.

6. Defendemos, respetamos y amamos los símbolos patrios, porque dichos símbolos, constituyen la representación simbólica de todo lo que somos como país. Representan la unidad e identidad nacional y constituyen un factor de primer nivel que ayuda a la cohesión interna de todos los peruanos.

7. Defendemos el territorio, el dominio marítimo y el espacio aéreo que los cubre, porque el territorio es el elemento esencial para la existencia del Estado. El territorio es el espacio físico donde se ubica la población, el dominio marítimo es la extensión del mar adyacente hasta las 200 millas y el espacio aéreo es el que cubre a ambos.

\section{DESARROLLO DE LA DEFENSA NACIONAL}

\section{Movilización y educación}

La defensa nacional es el organismo encargado de tratar y coordinar el trabajo de la defensa del país. La tarea principal consiste en aplicar la estrategia militar de defensa activa, organizar y ejecutar la movilización de defensa nacional del país, coordinar la relación entre la economía y los asuntos militares, entre el ejército y el gobierno y entre las fuerzas humanas y la garantía de materiales. Todo dirigido al trabajo de movilización para la defensa nacional, con el fin de fortalecer el poderío de la defensa nacional y elevar la capacidad de cambio entre los tiempos de paz y los de guerra.

En tiempos de paz, el Estado hace los preparativos relacionados con la movilización de las Fuerzas Armadas, del pueblo y de la economía nacional, integrándolos al programa y planes de desarrollo general del país. La movilización para la seguridad y defensa nacional es el conjunto de medidas y actividades destinadas a cambiar la estructura de las Fuerzas Armadas de tiempos de paz en otra de tiempos de guerra. La movilización de la economía nacional consiste en que el Estado aplica de manera organizada y planificada las medidas y actividades para el cambio de la estructura de la economía y los organismos correspondientes en tiempos de paz a la de los tiempos de guerra. La tarea principal de la movilización de la defensa es organizar la construcción y administración de proyectos de defensa, el sistema de comando, las comunicaciones, los sistemas de alarma y los campos para la dispersión, además de organizar la protección de las importantes metas económicas 
y educativas, además de la administración de los gastos y fondos de la defensa.

Todos los organismos estatales y las Fuerzas Armadas, los partidos políticos y las asociaciones civiles, las empresas, instituciones y ciudadanos deben realizar los preparativos de movilización conforme a las estipulaciones de la ley, y cumplir la tarea de movilización reglamentada después de que el Estado emita el decreto de movilización. Actualmente, según la orientación de combinar los tiempos de paz con los de guerra, lo militar con lo civil y el concepto de mantener reservas para los efectivos militares, se debe perfeccionar aún más el sistema de movilización para la defensa nacional, fortaleciendo su potencialidad, elevando su capacidad y promoviendo el continuo desarrollo de la movilización de la defensa nacional hacia la modernización. Aquí debe considerarse el desarrollo de la ciencia y tecnología de las universidades, centros nacionales de $\mathrm{I}+\mathrm{D}$, empresa privada y Fuerzas Armadas.

Sobre la educación en defensa nacional, nuestro país debe desarrollar el principio de combinar la educación regular con la intensiva, la general con la prioritaria y la teórica con la activa. La educación que se imparte a todos los ciudadanos respecto de la teoría, el espíritu, los conocimientos y las habilidades de la defensa nacional, debe orientarse a fortalecer la concepción y el dominio de los conocimientos sobre ella, a desarrollar el espíritu patriótico y a cumplir de manera consciente y responsable los deberes de la defensa nacional.

\section{Gastos para la defensa nacional}

Teóricamente los gastos para la defensa nacional incluyen aquellos destinados al sustento del personal, al mantenimiento de actividades y al armamento. Los gastos para el sustento del personal son utilizados esencialmente para el salario, la alimentación y los uniformes de los oficiales, soldados y empleados; los gastos para el mantenimiento de actividades se asignan principalmente al entrenamiento de las Fuerzas Armadas, la construcción y mantenimiento de obras y gastos de consumo corriente; los gastos en armamento se usan principalmente para la adquisición, el mantenimiento, el transporte y el almacenamiento de armas y equipos. Consideramos que los gastos para la investigación científica y experimentación militar deberían incrementarse.

\section{Industria de la defensa nacional}

En los países de economía fuerte se desarrolla la ciencia y tecnología de defensa nacional con el fundamental objetivo de satisfacer la necesidad básica de la defensa nacional, garantizar la producción y el suministro de las armas y los equipos militares y elevar el nivel de la modernización de la defensa nacional. Pero en el Perú, lejos de fortalecerlas, dichas industrias se han desmantelado.

Practicando la independencia y apoyándose en sus propias fuerzas, el Perú debe establecer una inversión adecuada, un sistema relativamente perfecto de la investigación de ciencia y tecnología con la industria de la defensa nacional, satisfaciendo básicamente la necesidad de la evolución de las Fuerzas Armadas, compuestas por las fuerzas terrestres, navales, y aéreas.

La industria, la ciencia y la tecnología militar para la defensa nacional deben buscar impulsar su desarrollo mediante la ciencia y la educación, utilizando plenamente las fuerzas científicas y tecnológicas de todo el país para desarrollar el estudio y la producción de tecnología militar, fortaleciendo la cooperación y el intercambio de la ciencia y la tecnología en defensa nacional con el extranjero, promoviendo el estudio y la fabricación de armamentos de alta y nueva tecnología y haciendo esfuerzos por ofrecer al ejército equipos y armas de avanzado 
funcionamiento, buena calidad y en series completas. Al mismo tiempo, impulsando con enorme esfuerzo la utilización pacífica de las técnicas de la industria militar, se deberán desarrollar prioritariamente las ramas de electrónica de uso civil, aeroespacial, aeronáutico y de barcos, materializando así la promoción recíproca entre las industrias militar y civil.

\section{Aspectos de ciencia, tecnología e industria para} la defensa nacional

Se debe tener en cuenta lo siguiente:

a. La ciencia y tecnología deben tener un desarrollo rápido. $\mathrm{El}$ ingreso total, el valor agregado industrial y los ingresos de venta de productos industriales de todo el año deben aumentar en comparación con el año anterior para beneficio del país.

b. Se debe hacer grandes progresos en la construcción de centrales hidroeléctricas, formando comparativamente un sistema completo de ciencia, tecnología e industria, con energía limpia y a bajo costo.

c. La navegación espacial para fines civiles debe hacer grandes progresos en los puntos tecnológicos cruciales para la construcción de cohetes.

d. Obtener nuevos progresos en la investigación y producción de bienes de uso civil en las universidades y que puedan servir para la defensa nacional.

e. Comenzar un vigoroso avance de la industria de construcción naval para aumentar su participación en el mercado mundial de la construcción naval, sobre todo considerando que sigue manteniendo una tendencia decreciente.

f. Crecer constantemente en la industria pilar de productos de uso civil para la industria militar.

g. Formación de profesionales en la cultura de la tecnología militar y civil.

\section{CONCLUSIÓN}

Relacionando la ciencia, la tecnología, la seguridad y la defensa nacional, las universidades, los institutos nacionales de $\mathrm{I}+\mathrm{D}$, las empresas privadas y nacionales y las Fuerzas Armadas deben conformar un sistema integrado en todos los campos de la actividad productiva de $\mathrm{I}+\mathrm{D}$, a fin de prepararse para un desarrollo continuo y sostenido que otorgue valor competitivo a los recursos naturales estratégicos. De ese modo estaremos en capacidad capaces de afrontar exitosamente con I+D las amenazas externas e internas sin afectar el bienestar de los peruanos. Asimismo se deberá dotar a este sistema de los recursos financieros necesarios, procedentes de un porcentaje determinado del PBI, de las exportaciones o del canon minero. 